\title{
A quark model study of $\psi(4260)$
}

\author{
R. Bruschini and P. González* \\ Departamento de Física Teórica-IFIC \\ Universidad de Valencia-CSIC \\ E-46100 Burjassot (Valencia), Spain. \\ E-mail: roberto.bruschini@ific.uv.es, pedro.gonzalez@uv.es
}

Following lattice results for the quark-antiquark static energy when mixing with an open flavor meson-meson configuration is considered, we build a a Born-Oppenheimer potential for $J^{P C}=$ $1^{--}$charmonium states below their first S- wave meson-meson threshold. We show that a good description of the mass and decay properties of the experimentally well established resonances in this spectral region, including conventional states and the unconventional $\psi(4260)$, is feasible.

XIII Quark Confinement and the Hadron Spectrum - Confinement2018

31 July - 6 August 2018

Maynooth University, Ireland

${ }^{*}$ Speaker. 


\section{Introduction}

The explanation of experimentally discovered charmonium states, that do not fit well in conventional quark model descriptions of heavy quarkonia, as for instance the ones provided by the Cornell [1] or the Godfrey-Isgur [2] models, is nowadays a theoretical challenge. Regarding unconventional isospin 0 states $\left(\chi_{c 1}(3872), \psi(4260) \ldots\right)$, see [3], the presence of close open flavor (charm) meson-meson thresholds may be playing an important role. As a matter of fact explanations involving meson-meson components in the form of either molecules, or tetraquarks -implicitly involving several molecular configurations-, or complementary configurations to the heavy quarkantiquark ones have been developed (for bibliographic reviews see [4]). Specifically the possible role played by the $D \bar{D}_{1}(2420)$ configuration in the description of $\psi(4260)$ has been emphasized by some authors, see for example [5].

In this talk we present an alternative explanation for $\psi(4260)$ based on a quark model description where the meson-meson degrees of freedom as well as the gluon ones are integrated out through an effective heavy quark-antiquark potential [?]. The form of this effective potential is guessed from lattice calculations of the quark-antiquark static energy when mixing with an open flavor meson-meson configuration is considered [7]. The contents of this presentation are organized as follows. In Section 2 we make a brief review of lattice results and use of a Born-Oppenheimer approximation to build a static potential for a $0^{-}\left(1^{--}\right)$charm $(c)$ - anticharm $(\bar{c})$ system below its corresponding first $S$ - wave meson-meson threshold. From it we obtain the spectrum and make a one to one assignment of the calculated states to the well established charmonium resonances below threshold. In Section 3 we center on the study of $\psi(4260)$, the only unconventional resonance in this energy region. We calculate its decay properties and compare them to existing data. Finally in Section 4 our main results and conclusions are summarized.

\section{2. $c \bar{c}$ Potential for $0^{-}\left(1^{--}\right)$states}

In order to construct a $Q \bar{Q}$ static potential implicitly incorporating the effect of meson-meson components we start from (unquenched) lattice results [7] for the energy of two static color sources ( $Q$ and $\bar{Q}$ ) when mixing of the $Q \bar{Q}$ configuration with the first static open flavor meson-meson one is taken into consideration. As a consequence of this mixing the $Q \bar{Q}$ static energy changes its radial dependence on the $Q-\bar{Q}$ distance. More precisely, at a certain energy below the meson-meson threshold the static energy dependence starts to deviate from a Cornell like form (corresponding to the case with only the $Q \bar{Q}$ configuration) becoming flat when reaching the threshold (henceforth we shall be only interested in this change; for the variation above threshold and its possible potential interpretation see [7], [8]).

Let us consider a $0^{-}\left(1^{--}\right) c \bar{c}$ configuration. Notice that the first static open flavor mesonmeson configuration is $D \bar{D}_{1}$ (including the degenerate $D \bar{D}_{1}(2420)$ and $D \bar{D}_{1}(2430)$ ) and not $D \bar{D}$ that has a lower threshold. The reason is that as $D \bar{D}$ would have orbital angular momentum 1 to get these quantum numbers the mesons would not be static as required. According to lattice results we expect $c \bar{c}$ and $D \bar{D}_{1}$ mixing starting at a certain energy $E_{s}=E_{t h}-\Delta$ where $E_{t h}$ is the threshold mass and $\Delta$ stands for the energy interval where mixing takes place. Hence we expect the $c-\bar{c}$ distance 
dependence of the static $c \bar{c}$ energy to deviate from the Cornell like form from $E_{s}$ to $E_{t h}$ where it becomes flat, signaling that the $c \bar{c}$ string has broken at this energy.

Following a Born-Oppenheimer approximation we identify this static energy dependence with a static potential. To take into account in a simple manner the gradual deviation from the Cornell like form we shall assume that at $E_{s}$ the potential reduces its slope (the one from the Cornell like form) to a constant value $s$ which is maintained up to $E_{t h}$ where it becomes 0 . This should be considered as an average approximation to the gradual decreasing of the slope that it is expected to really take place. Then the resulting potential in the energy interval $\left[0, m_{D \bar{D}_{1}}\right]$ with $m_{D \bar{D}_{1}}=$ $m_{D}+m_{\bar{D}_{1}}$ reads

$$
V_{\left[0, m_{D \bar{D}_{1}}\right]}(r)=\left\{\begin{array}{lr}
\sigma r-\frac{\zeta}{r} & r \leq r_{\Delta} \\
\left(m_{D \bar{D}_{1}}-m_{c}-m_{\bar{c}}-\Delta\right)+s\left(r-r_{\Delta}\right) & r_{\Delta} \leq r \leq\left(r_{\times}\right)_{\bar{D}_{1}} \\
m_{D \bar{D}_{1}}-m_{c}-m_{\bar{c}} & r \geq\left(r_{\times}\right)_{D \bar{D}_{1}}
\end{array}\right.
$$

where $r$ is the $c-\bar{c}$ distance and the parameters $\sigma$ and $\zeta$ stand for the string tension and the chromoelectric coulomb strength respectively. These two parameters altogether with the charm quark mass are fixed from the low lying bottomonium and charmonium spectra [8]. As for $r_{\Delta}$ and $\left(r_{\times}\right)_{D \bar{D}_{1}}$ they are defined by the continuity of the potential as

$$
\begin{gathered}
\sigma r_{\Delta}-\frac{\zeta}{r_{\Delta}}=E_{s}=E_{t h}-\Delta=m_{D \overline{D_{1}}}-m_{c}-m_{\bar{c}}-\Delta \\
E_{s}+s\left(\left(r_{\times}\right)_{D \bar{D}_{1}}-r_{\Delta}\right)=E_{t h}
\end{gathered}
$$

Regarding the value of $\Delta$ we should take into account that $\bar{D}_{1}^{0}(2420)$ has a width of about $30 \mathrm{MeV}$ and $\bar{D}_{1}^{0}(2430)$ has a much larger width. Henceforth we shall use the rather conservative (minimum) value $\Delta=60 \mathrm{MeV}$. With respect to the value of the slope $s$, we fix it by requiring that a bound state close below threshold appears as experimentally required by the presence of the unconventional $\psi(4260)$ resonance. It turns out that $s$ and $\Delta$ are correlated in the sense that an increasing of $\Delta$ can be compensated by an increasing of $s$ to get quite the same description for the bound state. The $0^{-}\left(1^{--}\right)$low lying spectrum obtained from this potential is shown in Table 1.

Notice that there is almost no difference between $V_{\left[0, m_{D \bar{D}_{1}}\right]}(r)$ and the Cornell like form $V_{C}(r)=$ $\sigma r-\frac{\zeta}{r}$ in the description of the (conventional) sates below $4200 \mathrm{MeV}$. On the contrary from this energy to threshold the use of $V_{\left[0, m_{D \bar{D}_{1}}\right]}(r)$ gives rise to the appearance of the $4 s_{\left[0, m_{D} \bar{D}_{1}\right]}$ and $3 d_{\left[0, m_{D \bar{D}_{1}}\right]}$ states with no correspondence at all with any conventional state from $V_{C}(r)$ (the $4 s$ state has a mass $\left.m_{\text {Cor }}(4 s)=4437 \mathrm{MeV}\right)$. This allows for the accommodation of $\psi(4260)$ as discussed in the next section.

3. $\psi(4260)$

In Table 1 the well established $\psi(4260)$ (different measurements of its mass go from 4222 $\mathrm{MeV}$ to $4284 \mathrm{MeV}$; the quoted average mass in [3] is $m_{\psi(4260)}=4230 \pm 8 \mathrm{MeV}$ ) has been assigned to the $4 s_{\left[0, m_{D \bar{D}_{1}}\right]}$ state with a calculated mass of $4261.5 \mathrm{MeV}$ although it is very probable that this 


\begin{tabular}{cccccc}
$J^{P C}$ & $\begin{array}{c}\text { States } \\
n l_{\left[0, m_{D \bar{D}_{1}}\right]}\end{array}$ & $\begin{array}{c}m_{\left[0, m_{D \bar{D}_{1}}\right]} \\
\mathrm{MeV}\end{array}$ & $\begin{array}{c}m_{P D G} \\
\mathrm{MeV}\end{array}$ & $\begin{array}{c}m_{\text {Cor }} \\
\mathrm{MeV}\end{array}$ & $\begin{array}{c}V_{C}(r) \text { States } \\
n l\end{array}$ \\
\hline $1^{--}$ & & & & \\
& $1 s_{\left[0, m_{D \bar{D}_{1}}\right]}$ & 3046.0 & $3096.916 \pm 0.011$ & 3046.0 & $1 s$ \\
& $2 s_{\left[0, m_{D \bar{D}_{1}}\right]}$ & 3632.1 & $3686.09 \pm 0.04$ & 3632.2 & $2 s$ \\
$1 d_{\left[0, m_{D \bar{D}_{1}}\right]}$ & 3743.4 & $3773.15 \pm 0.33$ & 3743.5 & $1 d$ \\
$3 s_{\left[0, m_{D \bar{D}_{1}}\right]}$ & 4061.0 & $4039 \pm 1$ & 4065.8 & $3 s$ \\
$2 d_{\left[0, m_{D \bar{D}_{1}}\right]}$ & 4136.4 & $4191 \pm 5$ & 4142.8 & $2 d$ \\
$4 s_{\left[0, m_{D \bar{D}_{1}}\right]}$ & 4261.5 & $4230 \pm 8$ & & \\
$3 d_{\left[0, m_{D \bar{D}_{1}}\right]}$ & 4277.3 & & &
\end{tabular}

Table 1: Calculated $0^{-}\left(1^{--}\right)$charmonium masses, $m_{\left[0, m_{D \bar{D}_{1}}\right]}$ from $V_{\left[0, m_{D \bar{D}_{1}}\right]}(r)$ with $\sigma=850 \mathrm{MeV} / \mathrm{fm}$, $\zeta=100 \mathrm{MeV} . \mathrm{fm}, m_{c}=1348.6 \mathrm{MeV}, m_{D \bar{D}_{1}}=4287 \mathrm{MeV}, \Delta=60 \mathrm{MeV}$ and $s=13 \mathrm{MeV} / \mathrm{fm}$. The spectral notation $n l_{\left[0, m_{D \bar{D}_{1}}\right]}$, where $n(l)$ indicates the principal (orbital angular momentum) quantum number, has been used for the states. Masses for experimental resonances, $m_{P D G}$, have been taken from [3]. Masses $m_{C o r}$ from the Cornell like form $V_{C}(r)=\sigma r-\frac{\zeta}{r}$, up to $m_{D \bar{D}_{1}}$, are also shown for comparison.

state mixes with the $3 d_{\left[0, m_{D \bar{D}_{1}}\right]}$ one giving rise a mass closer to the quoted experimental average. Under this assignment $\psi(4260)$ is an unconventional state coming out from the string breaking effect due to $D \bar{D}_{1}$ meson-meson components.

In our potential model these "molecular constituents" are embedded in the quark-antiquark $4 s_{\left[0, m_{D \bar{D}_{1}}\right]}$ wave function, as reflected by the value of its root mean square radius $\left\langle r^{2}\right\rangle^{\frac{1}{2}}=3.75 \mathrm{fm}$, much larger than for wave functions from $V_{C}(r)$ (for instance, $\left\langle r^{2}\right\rangle^{\frac{1}{2}}=1.55 \mathrm{fm}$ for the $4 s$ state with a mass of $4437 \mathrm{MeV})$. The non vanishing probability density at long distances for the $4 s_{\left[0, m_{D \bar{D}_{1}}\right]}$ state, say the non vanishing probability for the heavy quark and antiquark to be far apart, clearly indicates that string breaking has taken place (as a related consequence the probability density at the origin has been significantly reduced).

One could argue that it is not a big deal to get the mass of a state through the fixing of the free parameter $s$. Nonetheless once we have the wave function of $\psi(4260)$ we can calculate its decay properties and use their comparison to data as a stringent test of our effective description.

\section{$3.1 \psi(4260) \rightarrow e^{+} e^{-}$}

For conventional ${ }^{3} S_{1}$ bottomonium states below their corresponding $S$ - wave threshold the potential models $V_{C}(r)$ and $V_{\left[0, m_{B \overline{B_{1}}}\right]}(r)$, reproduce quite approximately the measured ratios of leptonic widths to $e^{+} e^{-}$(for explicit expressions for the ratios see for example [9]).

Regarding charmonium the calculated ratio $\frac{\Gamma_{2 s\left[0, m_{D} \bar{D}_{1}\right]} \rightarrow e^{+} e^{-}}{\Gamma_{\left.1 s_{\left[0, D_{D} \bar{D}_{1}\right.}\right] e^{+} e^{-}}}=\frac{\Gamma_{2 s \rightarrow e^{+} e^{-}}}{\Gamma_{1 s \rightarrow e^{+} e^{-}}}=0.5$ is a $15 \%$ off the experimental one $\left(\frac{\Gamma_{\psi(2 s) \rightarrow e^{+} e^{-}}}{\Gamma_{J / \psi \rightarrow e^{+} e^{-}}}\right)_{E x p}=0.42 \pm 0.02$.

Then, by assuming a similar quality for the calculated ratios involving the $4 s_{\left[0, m_{D \bar{D}_{1}}\right]}$ state we 
can use

$$
\frac{\Gamma_{\psi(4260) \rightarrow e^{+} e^{-}}}{\Gamma_{\psi(2 s) \rightarrow e^{+} e^{-}}} \simeq \frac{\Gamma_{4 s_{\left[0, m_{D} \bar{D}_{1}\right]} \rightarrow e^{+} e^{-}}}{\Gamma_{\left.2 s_{\left[0, m_{D} \bar{D}_{1}\right]}\right] e^{+} e^{-}}}=\frac{\left|R_{\psi(4260)}(0)\right|^{2}}{\left|R_{\psi(2 s)}(0)\right|^{2}} \frac{m_{\psi(2 s)}^{2}}{m_{\psi(4260)}^{2}}=2.4 \times 10^{-2}
$$

where $R_{\psi(4260)}(0) \simeq 1.5 \mathrm{fm}^{-\frac{3}{2}}$ and $R_{\psi(2 s)}(0) \simeq 8.3 \mathrm{fm}^{-\frac{3}{2}}$, altogether with the experimental measurement $\left(\Gamma_{\psi(2 s) \rightarrow e^{+} e^{-}}\right)_{E x p}=2.30 \pm 0.06 \mathrm{KeV}$ to predict an approximated leptonic decay width

$$
\Gamma_{\psi(4260) \rightarrow e^{+} e^{-}} \simeq 55.2 \pm 0.2 \mathrm{eV}
$$

Notice that this value is quite small as compared to $\left(\Gamma_{\psi(2 s) \rightarrow e^{+} e^{-}}\right)_{E x p}$ and other values for conventional states. This is a direct consequence of the lack of probability at the origin caused by screening expressed through the value of the radial wave function at the origin. Indeed our predicted $\Gamma_{\psi(4260) \rightarrow e^{+} e^{-}}$is in line with the experimental suppression of $S$ - wave $D \bar{D}_{1}$ production in $e^{+} e^{-}$annihilation. This has been explained as a consequence of approximated Heavy Quark Spin Symmetry, see references [10].

Unfortunately the $\psi(4260) \rightarrow e^{+} e$ width has not been measured separately for comparison. Instead we may use the experimentally known ratio

$$
\left(\frac{\Gamma_{\psi(4260) \rightarrow J / \psi \pi^{+} \pi^{-}} \Gamma_{\psi(4260) \rightarrow e^{+} e^{-}}}{\Gamma_{\psi(4260)}}\right)_{E x p}=9.2 \pm 1.0 \mathrm{eV}
$$

to guess from (3.2) the required branching ratio

$$
\frac{\Gamma_{\psi(4260) \rightarrow J / \psi \pi^{+} \pi^{-}}}{\Gamma_{\psi(4260)}} \simeq 0.17 \pm 0.03
$$

Then from the total measured width $\left(\Gamma_{\psi(4260)}\right)_{\text {Exp }}=55 \pm 19 \mathrm{MeV}$ we get

$$
\Gamma_{\psi(4260) \rightarrow J / \psi \pi^{+} \pi^{-}} \simeq 9 \pm 5 \mathrm{MeV}
$$

It is worthwhile to point out that the leptonic width would be smaller than the estimated value (3.2) if $\psi(4260)$ contained also some $3 d_{\left[0, m_{D \bar{D}_{1}}\right]}$ probability. This would make the branching ratio (3.4) and the decay width to $J / \psi \pi^{+} \pi^{-}$(3.5) to increase their estimated values.

For the sake of consistency $\Gamma_{\psi(4260) \rightarrow J / \psi \pi^{+} \pi^{-}}$should be reproduced from our quark model description. However, this calculation involving the emission of two gluons through intermediate hybrid states (see for instance [11]) is very uncertain due on the one hand to the lack of experimental knowledge of the hybrid states and on the other hand to the presumably pure convergence in this case of the multipole expansion used.

\subsection{E1 transitions}

For conventional charmonium (bottomonium) states below their corresponding $S$ - wave thresholds the potential models $V_{C}(r)$ and $V_{\left[0, m_{D \bar{D}_{1}}\right]}(r)\left(V_{\left[0, m_{B \bar{B}_{1}}\right]}(r)\right)$, give correctly the order of magnitude of the measured ratios of ${ }^{3} S_{1} \leftrightarrow{ }^{3} P_{1}$ dipole electric transitions from the same initial state or to the same final state (for explicit expressions for the ratios see for example [9] and references therein). 
More accurate results are obtained if the experimental masses of the states are used instead of the calculated ones.

By reasonably assuming the correct order of magnitude of the ratios when transitions from $4 s_{\left[0, m_{\left.D \bar{D}_{1}\right]}\right]}$ are involved we predict (for $\psi(2 s)$ and $\chi_{c 1}(1 p)$ the experimental masses are used; as for $\psi(4260)$ the calculated mass is taken since we do not consider mixing with the $3 d_{\left[0, m_{D \bar{D}_{1}}\right]}$ state)

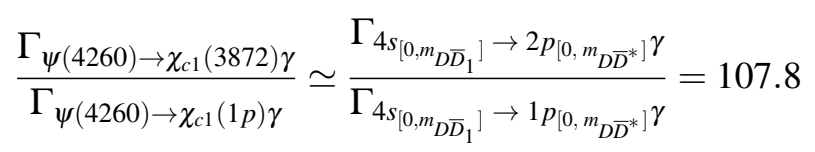

where for $\chi_{c 1}$ (3872) we have consistently used the unconventional wave function obtained in reference $[8]$ and

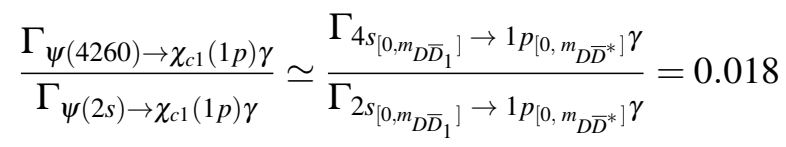

The first ratio (3.6) provides an explanation for the decay $\psi(4260) \rightarrow \chi_{c 1}$ (3872) $\gamma$ being seen against the not seen decay $\psi(4260) \rightarrow \chi_{c 1}(1 p) \gamma$. More quantitatively, we may use the second ratio (3.7) to predict from the experimental value $\left(\Gamma_{\psi(2 s) \rightarrow \chi_{c 1}(1 p) \gamma}\right)_{E x p}=29 \pm 1 \mathrm{KeV}$ a width

$$
\Gamma_{\psi(4260) \rightarrow \chi_{c 1}(1 p) \gamma} \simeq 0.506 \pm 0.017 \mathrm{KeV}
$$

Then from the first ratio we predict

$$
\Gamma_{\psi(4260) \rightarrow \chi_{c 1}(3872) \gamma} \simeq 54.6 \pm 1.9 \mathrm{KeV}
$$

We should keep in mind though that according to our assumption above these values of the widths should be considered as indicative of their order of magnitude and not as accurate predictions.

\section{$3.3 \psi(4260) \rightarrow D \bar{D}$}

Other issue about $\psi(4260)$ has to do with the experimental suppression of the $D \bar{D}$ decay mode (as compared to the case of conventional states like $\psi(3770)$ ) despite the fact that $\psi(4260)$ is above the $D \bar{D}$ threshold mass. In order to calculate this decay we rely on the ${ }^{3} P_{0}$ decay model [12] where the physical mechanism involved is related to the one we have used to take into account color screening in the potential (a $q \bar{q}$ created in the hadronic vacuum with $0^{++}$quantum numbers combines with $\bar{c} c$ giving rise to $D \bar{D}$ ). This model provides sensible results for the $D \bar{D}$ decay of the low lying conventional bottomonium and charmonium states with mass above the $D \bar{D}$ threshold. The explicit expression for the width can be easily obtained from [13]. Thus we get the ratio

$$
\frac{\Gamma_{\psi(4260) \rightarrow D \bar{D}}}{\Gamma_{\psi(3770) \rightarrow D \bar{D}}} \simeq \frac{\Gamma_{4 s_{\left[0, m_{D} \bar{D}_{1}\right]} \rightarrow D \bar{D}}}{\Gamma_{1 d_{\left[0, m_{D} \bar{D}_{1}\right]} \rightarrow D \bar{D}}}=7 \times 10^{-3}
$$

that explains the $D \bar{D}$ decay suppression for $\psi(4260)$ as compared to the conventional $\psi(3770)$ state. Quantitatively, using this ratio and the measured values $\left(\Gamma_{\psi(3770) \rightarrow D \bar{D}}\right)_{E x p}=25.6 \pm 0.8$ $\mathrm{MeV}$ and $\left(\Gamma_{\psi(4260)}\right)_{E x p}=55 \pm 19 \mathrm{MeV}$ we predict

$$
\Gamma_{\psi(4260) \rightarrow D \bar{D}} \simeq 0.18 \pm 0.01 \mathrm{MeV}
$$


and

$$
\frac{\Gamma_{\psi(4260) \rightarrow D \bar{D}}}{\left(\Gamma_{\psi(4260)}\right)_{E x p}} \simeq(3 \pm 2) \times 10^{-3}
$$

\section{Summary}

Following lattice indications and a Born-Oppenheimer approximation we have built a static quark-antiquark potential for the description of $0^{-}\left(1^{--}\right)$states below their first $S$ - wave mesonmeson threshold. The resulting spectrum contains conventional like states as well as unconventional ones implicitly incorporating meson-meson components. This allows for the theoretical accommodation of the experimentally well established resonance $\psi(4260)$ through its assignment to a calculated state. To check the viability of such an assignment we have calculated $e^{+} e^{-}, E 1$ and $D \bar{D}$ decay widths. Our results show full compatibility with existing data although more refined measurements would be needed for a more detailed comparison. Meanwhile we may tentatively conclude that $\psi(4260)$ may be described as an unconventional state coming out from the string breaking effect due to $D \bar{D}_{1}$ meson-meson components.

This work has been supported by Ministerio de Economía y Competitividad of Spain (MINECO) and EU Feder grant FPA2016-77177-C2-1-P and by SEV-2014-0398. R. B. acknowledges the Ministerio de Ciencia, Innovación y Universidades of Spain for a FPI fellowship.

\section{References}

[1] E. Eichten, K. Gottfried, T. Kinoshita, K. D. Lane and T. M. Yan, Phys. Rev. D 17, 3090 (1978); Phys. Rev. D 21, 203 (1980); E. J. Eichten, K. D. Lane and C. Quigg, Phys. Rev. D 69, 094019 (2004).

[2] S. Godfrey and N. Isgur, Phys. Rev. D 32, 189 (1985).

[3] M. Tanabashi et al. (Particle Data Group (PDG)), Phys. Rev. D 98, 030001 (2018).

[4] N. Brambilla et al., Eur. Phys. J. C 71, 1534 (2011); H-X. Chen, W. Chen, X. Liu and S-L. Zhu, Phys. Rep. 639, 1 (2016); R. F. Lebed, R. E. Mitchell and E. S. Swanson, Prog. Part. Nuc. Phys. 93, 143 (2017); F-K. Guo, C. Hanhart, U-G. Meissner, Q. Wang, Q. Zhao, B-S. Zou, Rev. Mod. Phys. 90, 015004 (2018); S. L. Olsen, T. Skwarnicki and D. Zieminska, Rev.Mod.Phys. 90, 015003. (2018).

[5] J. L. Rosner, Phys. Rev. D 74, 076006 (2006); G. J. Ding, Phys. Rev. D 79, 014001 (2009); Q. Wang, C. Hanhart and Q. Zhao, Phys. Rev. Lett. 111, 132003 (2013); F. K. Guo, C. Hanhart, U. G. Meissner, Q. Wang and Q. Zhao, Phys. Lett. B 725, 127 (2013); W. Qin, S-R. Xue and Q. Zhao, Phys. Rev. D 94, 054035 (2016).

[6] R.Bruschini and P. González, Phys. Rev. C 99 , 045205 (2019).

[7] G. S. Bali, H. Neff, T. Düssel, T. Lippert and K. Schilling (SESAM Collaboration), Phys. Rev. D 71, 114513 (2005).

[8] P. González, J.Phys. G 41, 095001 (2014); P. González, Phys. Rev. D 92, 014017 (2015).

[9] E. Eichten, S. Godfrey, H. Mahlke, and J. L. Rosner, Rev. Mod. Phys. 80, 1161 (2008).

[10] X. Li and M. B. Voloshin, Phys. Rev. D 88, 034012 (2013); Q. Wang, M. Cleven, F-K. Guo, C. Hanhart, U-G. Meissner, X-G. Wu and Q. Zhao, Phys. Rev. D 89, 034001 (2014). 
[11] Y-P. Kuang, Int. J. Mod. Phys. A 24 S1, 327 (2009).

[12] A. Le Yaouanc, L. Oliver, O. Pène and J. C. Raynal in "Hadron transitions in the quark model", Gordon and Breach Science Publishers 1988; Phys. Rev. D 8, 2223 (1973); A. Le Yaouanc, L. Oliver, O. Pene and J-C. Raynal, Phys. Lett. B 71, 397 (1977).

[13] S. Ono, Phys. Rev. D 23, 1118 (1981). 